International Journal of Pure and Applied Mathematics

Volume 89 No. 1 2013, 9-18

ISSN: 1311-8080 (printed version); ISSN: 1314-3395 (on-line version)

url: http://www.ijpam.eu

doi: http://dx.doi.org/10.12732/ijpam.v89i1.2

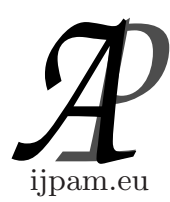

\title{
INTEGRAL MEAN ESTIMATES FOR POLYNOMIALS WITH RESTRICTED ZEROS
}

\author{
N.A. Rather ${ }^{1}$, Suhail Gulzar ${ }^{2} \S$ \\ ${ }^{1,2}$ Department of mathematics \\ University of Kashmir \\ Harzarbal, Sringar 190006, INDIA
}

Abstract: Let $P(z)$ be a polynomial of degree $n$ having all its zeros in $|z| \leq k$, where $k \leq 1$, then for each $r>0, p>1, q>1$ with $p^{-1}+q^{-1}=1$, it was proved by Aziz and Ahemad [2]:

$$
n\left\{\int_{0}^{2 \pi}\left|P\left(e^{i \theta}\right)\right|^{r} d \theta\right\}^{\frac{1}{r}} \leq\left\{\int_{0}^{2 \pi}\left|1+k e^{i \theta}\right|^{q r} d \theta\right\}^{\frac{1}{q r}}\left\{\int_{0}^{2 \pi}\left|P^{\prime}\left(e^{i \theta}\right)\right|^{p r} d \theta\right\}^{\frac{1}{p r}} .
$$

In this paper, we establish some refinements and generalizations of above and some known polynomial inequalities concerning the polar derivative of a polynomial with restricted zeros.

AMS Subject Classification: 30C10, 30A10, 41A17

Key Words: polynomials, inequalities in the complex domain, polar derivative

\section{Introduction and Statement of the Results}

Let $P(z)$ be a polynomial of degree $n$. It was shown by Turán [12] that if $P(z)$ has all its zeros in $|z| \leq 1$, then

$$
n \max _{|z|=1}|P(z)| \leq 2 \max _{|z|=1}\left|P^{\prime}(z)\right| \text {. }
$$

Inequality (1.1) is best possible with equality holds for $P(z)=\alpha z^{n}+\beta$, where

Received: March 29, 2013

(C) 2013 Academic Publications, Ltd.

$\S_{\text {Correspondence author }}$ url: www.acadpubl.eu 
$|\alpha|=|\beta| \neq 0$.

As an extension of (1.1), Malik [9] proved that if $P(z)$ is a polynomial of degree $n$ having all its zeros in $|z| \leq k$, where $k \leq 1$, then

$$
n \max _{|z|=1}|P(z)| \leq(1+k) \max _{|z|=1}\left|P^{\prime}(z)\right| .
$$

Equality in (1.2) holds for $P(z)=(z+k)^{n}$, where $k \leq 1$.

On the other hand, for the class of polynomials $P(z)=a_{n} z^{n}+\sum_{j=\mu}^{n} a_{n-j} z^{n-j}$, $1 \leq \mu \leq n$, of degree $n$ having all their zeros in $|z| \leq k, k \leq 1$, Aziz and Shah [6] proved that

$$
n \max _{|z|=1}|P(z)| \leq\left(1+k^{\mu}\right) \max _{|z|=1}\left|P^{\prime}(z)\right|-\frac{n}{k^{n-\mu}} \min _{|z|=k}|P(z)| .
$$

Malik [10] obtained a generalization of (1.1) in the sense that the left-hand side of (1.1) is replaced by a factor involving the integral mean of $|P(z)|$ on $|z|=1$. In fact, he proved that if $P(z)$ is a polynomial of degree $n$ having all its zeros in $|z| \leq 1$, then for each $q>0$,

$$
n\left\{\int_{0}^{2 \pi}\left|P\left(e^{i \theta}\right)\right|^{q} d \theta\right\}^{\frac{1}{q}} \leq\left\{\int_{0}^{2 \pi}\left|1+e^{i \theta}\right|^{q} d \theta\right\}_{|z|=1}^{\frac{1}{q}} \max _{\left|P^{\prime}(z)\right| .}
$$

The corresponding extension of (1.2), which is a generalization of (1.4), was obtained by Aziz [1] who proved that if $P(z)$ is a polynomial of degree $n$ having all its zeros in $|z| \leq k$, where $k \leq 1$, then for each $q \geq 0$

$$
n\left\{\int_{0}^{2 \pi}\left|P\left(e^{i \theta}\right)\right|^{q} d \theta\right\}^{\frac{1}{q}} \leq\left\{\int_{0}^{2 \pi}\left|1+k e^{i \theta}\right|^{q} d \theta\right\}^{\frac{1}{q}} \max _{|z|=1}\left|P^{\prime}(z)\right| .
$$

Inequality (1.5) reduces to the inequality (1.2) by letting $q \rightarrow \infty$.

As a generalization of (1.5), Aziz and Ahemad [2] proved that if $P(z)$ is a polynomial of degree $n$ having all its zeros in $|z| \leq k$, where $k \leq 1$, then for each $r>0, p>1, q>1$ with $p^{-1}+q^{-1}=1$,

$$
n\left\{\int_{0}^{2 \pi}\left|P\left(e^{i \theta}\right)\right|^{r} d \theta\right\}^{\frac{1}{r}} \leq\left\{\int_{0}^{2 \pi}\left|1+k e^{i \theta}\right|^{q r} d \theta\right\}^{\frac{1}{q r}}\left\{\int_{0}^{2 \pi}\left|P^{\prime}\left(e^{i \theta}\right)\right|^{p r} d \theta\right\}^{\frac{1}{p r}}
$$

Let $D_{\alpha} P(z)$ denote the polar derivative of a polynomial $P(z)$ of degree $n$ with respect to a point $\alpha \in \mathbb{C}$, then (see [11])

$$
D_{\alpha} P(z)=n P(z)+(\alpha-z) P^{\prime}(z) .
$$


The polynomial $D_{\alpha} P(z)$ is of degree at most $n-1$ and it generalizes the ordinary derivative in the sense that

$$
\lim _{\alpha \rightarrow \infty} \frac{D_{\alpha} P(z)}{\alpha}=P^{\prime}(z)
$$

uniformly with respect to $z$ for $|z| \leq R, R>0$.

As an extension of (1.2) to the polar derivative, Aziz and Rather [3] proved that if all the zeros of $P(z)$ lie in $|z| \leq k$, where $k \leq 1$, then for every real or complex number $\alpha$ with $|\alpha| \geq k$,

$$
n(|\alpha|-k) \max _{|z|=1}|P(z)| \leq(1+k) \max _{|z|=1}\left|D_{\alpha} P(z)\right| .
$$

For the class of Lacunary polynomials $P(z)=a_{n} z^{n}+\sum_{\nu=\mu}^{n} a_{n-\nu} z^{n-\nu}, 1 \leq$ $\mu \leq n$, of degree $n$ having all their zeros in $|z| \leq k$, where $k \leq 1$, Aziz and Rather [4] also proved that if $\alpha$ is any real or complex number with $|\alpha| \geq S_{\mu}$ then

$$
n\left(|\alpha|-S_{\mu}\right) \max _{|z|=1}|P(z)| \leq\left(1+S_{\mu}\right) \max _{|z|=1}\left|D_{\alpha} P(z)\right|,
$$

where

$$
S_{\mu}=\frac{n\left|a_{n}\right| k^{2 \mu}+\mu\left|a_{n-\mu}\right| k^{\mu-1}}{n\left|a_{n}\right| k^{\mu-1}+\mu\left|a_{n-\mu}\right|} .
$$

In view of (1.9), one can easily verify that the inequality (1.8) is a generalization as well as a refinement of inequality (1.7).

In this paper, we consider the class of polynomials

$$
P(z)=a_{n} z^{n}+\sum_{\nu=\mu}^{n} a_{n-\nu} z^{n-\nu}, \quad 1 \leq \mu \leq n,
$$

having all its zeros in $|z| \leq k$, where $k \leq 1$ and establish some generalizations of inequalities (1.1),(1.2),(1.7),(1.5) and (1.8).

In this direction, we present the following interesting results which yields (1.8) as a special case.

Theorem 1.1. If $P(z)=a_{n} z^{n}+\sum_{\nu=\mu}^{n} a_{n-\nu} z^{n-\nu}, 1 \leq \mu \leq n$, is a polynomial of degree $n$ having all its zeros in $|z| \leq k$, where $k \leq 1$, then for all real or complex numbers $\alpha, \lambda$ with $|\alpha| \geq A_{\mu},|\lambda| \leq 1$ and for each $r>0, p>1, q>1$ with $p^{-1}+q^{-1}=1$,

$$
n\left(|\alpha|-A_{\mu}\right)\left\{\int_{0}^{2 \pi}\left|P\left(e^{i \theta}\right)+\frac{\lambda m e^{i n \theta}}{k^{n}}\right|^{r} d \theta\right\}^{\frac{1}{r}} \leq\left\{\int_{0}^{2 \pi}\left|1+A_{\mu} e^{i \theta}\right|^{p r} d \theta\right\}^{\frac{1}{p r}}
$$




$$
\times\left\{\int_{0}^{2 \pi}\left|D_{\alpha} P\left(e^{i \theta}\right)+\frac{\lambda \alpha m n e^{i(n-1) \theta}}{k^{n}}\right|^{q r} d \theta\right\}^{\frac{1}{q r}}
$$

where $m=\min _{|z|=k}|P(z)|$ and

$$
A_{\mu}=\frac{n\left(\left|a_{n}\right|-\frac{m}{k^{n}}\right) k^{2 \mu}+\mu\left|a_{n-\mu}\right| k^{\mu-1}}{n\left(\left|a_{n}\right|-\frac{m}{k^{n}}\right) k^{\mu-1}+\mu\left|a_{n-\mu}\right|} .
$$

If we divide two sides of inequality (1.10) by $|\alpha|$ and then letting $|\alpha| \rightarrow \infty$, we obtain the following generalization of inequality (1.3).

Corollary 1.2. If $P(z)=a_{n} z^{n}+\sum_{\nu=\mu}^{n} a_{n-\nu} z^{n-\nu}, 1 \leq \mu \leq n$, is a polynomial of degree $n$ having all its zeros in $|z| \leq k$, where $k \leq 1$, then for every real or complex number $\lambda$ with $|\lambda| \leq 1$ and for each $r>0, p>1, q>1$ with $p^{-1}+q^{-1}=1$,

$$
\begin{aligned}
n\left\{\int_{0}^{2 \pi} \mid P\left(e^{i \theta}\right)+\right. & \left.\left.\frac{\lambda m e^{i n \theta}}{k^{n}}\right|^{r} d \theta\right\}^{\frac{1}{r}} \leq\left\{\int_{0}^{2 \pi}\left|1+A_{\mu} e^{i \theta}\right|^{p r} d \theta\right\}^{\frac{1}{p r}} \\
& \times\left\{\int_{0}^{2 \pi} \mid P^{\prime}\left(e^{i \theta}\right)+\frac{\left.\lambda m n e^{i(n-1) \theta}\right|^{q r}}{k^{n}} d \theta\right\}^{\frac{1}{q r}},
\end{aligned}
$$

where $m=\min _{|z|=k}|P(z)|$ and $A_{\mu}$ is given by (1.11).

Remark 1.3. If we take $\lambda=0$ in (1.12), then we get a refinement of inequality (1.6).

Letting $p \rightarrow \infty$, so that $q \rightarrow 1$ in (1.10), we obtain the following result.

Corollary 1.4. If $P(z)=a_{n} z^{n}+\sum_{\nu=\mu}^{n} a_{n-\nu} z^{n-\nu}, 1 \leq \mu \leq n$, is a polynomial of degree $n$ having all its zeros in $|z| \leq k$, where $k \leq 1$, then for all real or complex numbers $\alpha, \lambda$ with $|\alpha| \geq A_{\mu},|\beta| \leq 1$ and for each $r>0$,

$$
\begin{aligned}
& n\left(|\alpha|-A_{\mu}\right)\left\{\int_{0}^{2 \pi}\left|P\left(e^{i \theta}\right)+\frac{\lambda m e^{i n \theta}}{k^{n}}\right|^{r} d \theta\right\}^{\frac{1}{r}} \\
& \leq\left(1+A_{\mu}\right)\left\{\int_{0}^{2 \pi}\left|D_{\alpha} P\left(e^{i \theta}\right)+\frac{\lambda m n \alpha e^{i(n-1) \theta}}{k^{n}}\right|^{r} d \theta\right\}^{\frac{1}{r}},
\end{aligned}
$$

where $m=\min _{|z|=k}|P(z)|$ and $A_{\mu}$ is given by (1.11). 
By Lemma 2.4, we have $A_{\mu} \leq k^{\mu}$ and therefore, it can be verified that for $p>1$ and $r>0$,

$$
\int_{0}^{2 \pi}\left|1+A_{\mu} e^{i \theta}\right|^{p r} d \theta \leq \int_{0}^{2 \pi}\left|1+k^{\mu} e^{i \theta}\right|^{p r} d \theta .
$$

In view of inequality (1.14), we get the following result.

Corollary 1.5. If $P(z)=a_{n} z^{n}+\sum_{\nu=\mu}^{n} a_{n-\nu} z^{n-\nu}, 1 \leq \mu \leq n$, is a polynomial of degree $n$ having all its zeros in $|z| \leq k$, where $k \leq 1$, then for all real or complex numbers $\alpha, \lambda$ with $|\alpha| \geq A_{\mu},|\lambda| \leq 1$ and for each $r>0$, $p>1, q>1$ with $p^{-1}+q^{-1}=1$,

$$
\begin{gathered}
n\left(|\alpha|-A_{\mu}\right)\left\{\int_{0}^{2 \pi}\left|P\left(e^{i \theta}\right)+\frac{\lambda m e^{i n \theta}}{k^{n}}\right|^{r} d \theta\right\}^{\frac{1}{r}} \leq\left\{\int_{0}^{2 \pi}\left|1+k^{\mu} e^{i \theta}\right|^{p r} d \theta\right\}^{\frac{1}{p r}} \\
\times\left\{\int_{0}^{2 \pi}\left|D_{\alpha} P\left(e^{i \theta}\right)+\frac{\lambda \alpha m n e^{i(n-1) \theta}}{k^{n}}\right|^{q r} d \theta\right\}^{\frac{1}{q r}}
\end{gathered}
$$

where $m=\operatorname{Min}_{|z|=k}|P(z)|$ and $A_{\mu}$ is given by (1.11).

\section{Some Lemmas}

For the proof of theorem 1.1, we need the following lemmas:

Lemma 2.1. If $P(z)=a_{n}+\sum_{j=\mu}^{n} a_{n-j} z^{n-j}, 1 \leq \mu \leq n$, is a polynomial of degree $n$ having all its zeros in $|z| \leq k, k \leq 1$ and $Q(z)=z^{n} \overline{P(1 / \bar{z})}$, then for $|z|=1$

$$
\left|Q^{\prime}(z)\right| \leq S_{\mu}\left|P^{\prime}(z)\right|
$$

where $S_{\mu}$ is given by (1.9).

The above lemma is due to Aziz and Rather [5].

Lemma 2.2. If $P(z)=\sum_{j=1}^{n} a_{j} z^{j}$ is a polynomial of degree $n$ having all its zeros in $|z| \leq k, k \leq 1$ and $m=\min _{|z|=k}|P(z)|$, then

$$
\left|a_{n}\right|>\frac{m}{k^{n}} .
$$


Proof. By hypothesis all the zeros of $P(z)$ lie in $|z| \leq k$. If $P(z)$ has a zero on $|z|=k$, then $m=0$ and the result holds trivially. So, we assume $P(z)$ has all its zeros in $|z|<k, k \leq 1$, so that $m>0$. If $Q(z)=z^{n} \overline{P(1 / \bar{z})}$, then $Q(z)$ has no zero in $|z| \leq 1 / k$. By Minimum Modulus Principle

$$
|Q(z)| \geq \min _{|z|=1 / k}|Q(z)| \text { for }|z| \leq 1 / k, \text { where } 1 / k \geq 1
$$

which in particular gives,

$$
\left|a_{n}\right|=|Q(0)|>\frac{1}{k^{n}} \min _{|z|=k}|P(z)| .
$$

This completes the proof of Lemma 2.2..

Lemma 2.3. The function

$$
S_{\mu}(x)=\frac{n x k^{2 \mu}+\mu\left|a_{n-\mu}\right| k^{\mu-1}}{n x k^{\mu-1}+\mu\left|a_{n-\mu}\right|},
$$

where $k \leq 1$ and $\mu \geq 1$, is a non-increasing function of $x$.

Proof. The proof follows by considering the first derivative test for $S_{\mu}(x)$.

We also need following Lemma.

Lemma $2.4([7])$. If $P(z)=a_{n} z^{n}+\sum_{j=\mu}^{n} a_{n-j} z^{n-j}, 1 \leq \mu \leq n$, is a polynomial of degree $n$ having all its zeros in $|z| \leq k, k \leq 1$, then

$$
A_{\mu} \leq k^{\mu}
$$

where $A_{\mu}$ is defined in (1.11).

\section{Proof of Theorem}

Proof of Theorem 1.1. Let $m=\min _{|z|=k}|P(z)|$. By hypothesis $P(z)=$ $a_{n} z^{n}+\sum_{j=\mu}^{n} a_{n-j} z^{n-j}, 1 \leq \mu \leq n$, has all its zeros in $|z| \leq k$, where $k \leq 1$. We show $F(z)=P(z)+\frac{\lambda m z^{n}}{k^{n}}$ has all its zeros in $|z| \leq k$ for every $\lambda$ with $|\lambda| \leq 1$. This is obvious if $m=0$ that is, if $P(z)$ has a zero on $|z|=k$. Hence, we suppose that all the zeros of $P(z)$ lie in $|z|<k, k \leq 1$, so that $m>0$. Now $m \leq|P(z)|$ for $|z|=k$, therefore if $\lambda$ is any complex number such that $|\lambda|<1$, then

$$
\left|\frac{m \lambda z^{n}}{k^{n}}\right|<|P(z)| \text { for }|z|=k .
$$


Since all the zeros of $P(z)$ lie in $|z|<k$, it follows by Rouche's theorem that all the zeros of

$$
F(z)=P(z)+\frac{m \lambda z^{n}}{k^{n}}=\left(a_{n}+\frac{\lambda m}{k^{n}}\right) z^{n}+\sum_{j=\mu}^{n} a_{n-j} z^{n-j}
$$

also lie in $|z|<k, k \leq 1$.

Let $G(z)=z^{n} \overline{F(1 / \bar{z})}=z^{n} \overline{P(1 / \bar{z})}+\frac{m \bar{\lambda}}{k^{n}}$ then it can be easily verified for $|z|=1$,

$$
\left|F^{\prime}(z)\right|=\left|n G(z)-z G^{\prime}(z)\right| \text { and }\left|G^{\prime}(z)\right|=\left|n F(z)-z F^{\prime}(z)\right| .
$$

Applying Lemma 2.1 to the polynomial $F(z)$, we get for $|z|=1$,

$$
S_{\mu}^{\prime}\left|F^{\prime}(z)\right| \geq\left|G^{\prime}(z)\right|
$$

where

$$
S_{\mu}^{\prime}=\frac{n\left|a_{n}+\frac{m \lambda}{k^{n}}\right| k^{2 \mu}+\mu\left|a_{n-\mu}\right| k^{\mu-1}}{n\left|a_{n}+\frac{m \lambda}{k^{n}}\right| k^{\mu-1}+\mu\left|a_{n-\mu}\right|} .
$$

Since for every $\lambda$ with $|\lambda|<1$, we have by Lemma 2.2 ,

$$
\left|a_{n}+\frac{m \lambda}{k^{n}}\right| \geq\left|a_{n}\right|-\frac{m|\lambda|}{k^{n}} \geq\left|a_{n}\right|-\frac{m}{k^{n}},
$$

therefore it follows from Lemma 2.3, (3.3) and (3.4) that for every $\lambda$ with $|\lambda|<1$

$$
\begin{aligned}
S_{\mu}^{\prime} & =\frac{n\left|a_{n}+\frac{m \lambda}{k^{n}}\right| k^{2 \mu}+\mu\left|a_{n-\mu}\right| k^{\mu-1}}{n\left|a_{n}+\frac{m \lambda}{k^{n}}\right| k^{\mu-1}+\mu\left|a_{n-\mu}\right|} \\
& \leq \frac{n\left(\left|a_{n}\right|-\frac{m}{k^{n}}\right) k^{2 \mu}+\mu\left|a_{n-\mu}\right| k^{\mu-1}}{n\left(\left|a_{n}\right|-\frac{m}{k^{n}}\right) k^{\mu-1}+\mu\left|a_{n-\mu}\right|}=A_{\mu}
\end{aligned}
$$

Using (3.1) and (3.5) in (3.2), we obtain

$$
A_{\mu}\left|F^{\prime}(z)\right| \geq\left|n F(z)-z F^{\prime}(z)\right| \text { for }|z|=1 .
$$

This gives for every real or complex number $\alpha$ with $|\alpha| \geq A_{\mu}$, we have

$$
\begin{aligned}
\left|D_{\alpha} F(z)\right| & =\left|n F(z)+(\alpha-z) F^{\prime}(z)\right| \\
& \geq|\alpha|\left|F^{\prime}(z)\right|-\left|n F(z)-z F^{\prime}(z)\right| \\
& \geq\left(|\alpha|-A_{\mu}\right)\left|F^{\prime}(z)\right| \quad \text { for } \quad|z|=1 .
\end{aligned}
$$


Replacing $F(z)$ by $P(z)+\frac{\lambda m z^{n}}{k^{n}}$, we obtain for $|z|=1$,

$$
\left|D_{\alpha} P(z)+\frac{\lambda \alpha m n z^{n-1}}{k^{n}}\right| \geq\left(|\alpha|-A_{\mu}\right)\left|P^{\prime}(z)+\frac{\lambda \alpha m n z^{n-1}}{k^{n}}\right| .
$$

Since $F(z)$ has all its zeros in $|z| \leq k \leq 1$, it follows by Gauss-Lucas theorem that all the zeros of $F^{\prime}(z)$ also lie in $|z| \leq k \leq 1$. This shows that the polynomial

$$
z^{n-1} \overline{F^{\prime}(1 / \bar{z})} \equiv n G(z)-z G^{\prime}(z)
$$

has all its zeros in $|z| \geq(1 / k) \geq 1$. Therefore, it follows from (3.6) that the function

$$
w(z)=\frac{z G^{\prime}(z)}{A_{\mu}\left(n G(z)-z G^{\prime}(z)\right)}
$$

is analytic for $|z| \leq 1$ and $|w(z)| \leq 1$ for $|z|=1$. Furthermore, $w(0)=0$. Thus the function $1+A_{\mu} w(z)$ is subordinate to the function $1+A_{\mu} z$ for $|z| \leq 1$. Hence by a well known property of subordination [8], we have for each $r>0$,

$$
\int_{0}^{2 \pi}\left|1+A_{\mu} w\left(e^{i \theta}\right)\right|^{r} d \theta \leq \int_{0}^{2 \pi}\left|1+A_{\mu} e^{i \theta}\right|^{r} d \theta
$$

Now

$$
1+A_{\mu} w(z)=\frac{n G(z)}{n G(z)-z G^{\prime}(z)}
$$

and

$$
\left|F^{\prime}(z)\right|=\left|z^{n-1} \overline{F^{\prime}(1 / \bar{z})}\right|=\left|n G(z)-z G^{\prime}(z)\right| \text { for } \quad|z|=1,
$$

therefore for $|z|=1$,

$$
n|G(z)|=\left|1+A_{\mu} w(z)\right|\left|n G(z)-z G^{\prime}(z)\right|=\left|1+A_{\mu} w(z)\right|\left|F^{\prime}(z)\right| .
$$

Equivalently for $|z|=1$ :

$$
n\left|z^{n} \overline{P(1 / \bar{z})}+\frac{\bar{\lambda} m}{k^{n}}\right|=\left|1+A_{\mu} w(z)\right|\left|P^{\prime}(z)+\frac{\lambda \alpha m n z^{n-1}}{k^{n}}\right|,
$$

which implies for $|z|=1$,

$$
n\left|P(z)+\frac{\lambda m z^{n}}{k^{n}}\right|=\left|1+A_{\mu} w(z)\right|\left|P^{\prime}(z)+\frac{\lambda \alpha m n z^{n-1}}{k^{n}}\right| .
$$


From (3.8) and (3.10), we deduce that for each $r>0$,

$$
\begin{aligned}
n^{r}\left(|\alpha|-A_{\mu}\right)^{r} & \int_{0}^{2 \pi}\left|P\left(e^{i \theta}\right)+\frac{\lambda m e^{i n \theta}}{k^{n}}\right|^{r} d \theta \\
& \leq \int_{0}^{2 \pi}\left|1+A_{\mu} w\left(e^{i \theta}\right)\right|^{r}\left|D_{\alpha} P\left(e^{i \theta}\right)+\frac{\lambda \alpha m n e^{i(n-1) \theta}}{k^{n}}\right|^{r} d \theta .
\end{aligned}
$$

This gives with the help of (3.9) and using Hölder's inequality for $p>1, q>1$ with $p^{-1}+q^{-1}=1$,

$$
\begin{aligned}
n^{r}\left(|\alpha|-A_{\mu}\right)^{r} \int_{0}^{2 \pi} \mid & P\left(e^{i \theta}\right)+\left.\frac{\lambda m e^{i n \theta}}{k^{n}}\right|^{r} d \theta \leq\left(\int_{0}^{2 \pi}\left|1+A_{\mu} e^{i \theta}\right|^{p r} d \theta\right)^{1 / p} \\
& \times\left(\int_{0}^{2 \pi}\left|D_{\alpha} P\left(e^{i \theta}\right)+\frac{\lambda \alpha m n e^{i(n-1) \theta}}{k^{n}}\right|^{q r} d \theta\right)^{1 / q}
\end{aligned}
$$

equivalently

$$
\begin{gathered}
n\left(|\alpha|-A_{\mu}\right)\left\{\int_{0}^{2 \pi}\left|P\left(e^{i \theta}\right)+\frac{\lambda m e^{i n \theta}}{k^{n}}\right|^{r} d \theta\right\}^{r} \leq\left\{\int_{0}^{2 \pi}\left|1+A_{\mu} e^{i \theta}\right|^{p r} d \theta\right\}^{1 / p r} \\
\times\left\{\int_{0}^{2 \pi}\left|D_{\alpha} P\left(e^{i \theta}\right)+\frac{\lambda \alpha m n e^{i(n-1) \theta}}{k^{n}}\right|^{q r} d \theta\right\}^{1 / q r} .
\end{gathered}
$$

This completes the proof of theorem 1.1.

\section{Acknowledgments}

The work is supported by Council of Scientific and Industrial Research, New Delhi, under grant F.No. 09/251(0047)/2012-EMR-I.

\section{References}

[1] A. Aziz, Integral mean estimates polynomials with restricted zeros, J. Approx. Theory, 55 (1988), 232-239. 
[2] A. Aziz and N. Ahemad, Integral mean estimates for polynomials whose zeros are within a circle, Glas. Mat. Ser. III, 31 (1996), 229-237.

[3] A. Aziz and N. A. Rather, A refinement of a theorem of Paul Turan concerning polynomials. Math Ineq. Appl., 1, 231-238 (1998).

[4] A. Aziz and N. A. Rather, Inequalities for the polar derivative of a polynomial with restricted zeros, Math. Balk., 17 (2003), 15-28.

[5] A. Aziz and N. A. Rather, Some Zygmund type $L^{q}$ inequalities for polynomials, J. Math. Anal. Appl., 289 (2004) 14-29.

[6] A. Aziz and W. M. Shah, An integral mean estimate for polynomial, Indian J. Pure Appl. Math., 28 (1997) 1413-1419.

[7] K. K. Dewan, Naresh Singh, Abdullah Mir, Extensions of some polynomial inequalities to the polar derivative, J. Math. Anal. Appl., 352 (2009) 807815.

[8] E. Hille, Analytic function theory, Vol. II, Ginn and Company, New York, Toronto, 1962.

[9] M. A. Malik, On the derivative of a polynomial, J. Lond. Math. Soc., Second Series 1 (1969), 57-60.

[10] M. A. Malik, An integral mean estimates for polynomials, Proc. Amer. Math. Soc., 91 (1984), 281-284.

[11] M. Marden, Geometry of Polynomials, Math. Surveys No. 3, Amer. Math. Soc. Providence R. I. 1966.

[12] P. Turan, Uber die Ableitung von Polynomen, Compos. Math., 7 (1939), 89-95 (German). 